\title{
Les bruits de la bataille et le silence des archives : cinq réflexions et un épilogue
}

\section{Helmut Lethen}

Traducteur : Françoise Bornemann

\section{(2) OpenEdition Journals}

Édition électronique

URL : https://journals.openedition.org/rbnu/1712

DOl : $10.4000 /$ rbnu. 1712

ISSN : 2679-6104

\section{Éditeur}

Bibliothèque nationale et universitaire de Strasbourg

\section{Édition imprimée}

Date de publication : 1 novembre 2014

Pagination : 102-109

ISBN : 9782859230548

ISSN : 2109-2761

\section{Référence électronique}

Helmut Lethen, « Les bruits de la bataille et le silence des archives : cinq réflexions et un épilogue », La Revue de la BNU [En ligne], 10 | 2014, mis en ligne le 01 novembre 2014, consulté le 18 mai 2021. URL: http://journals.openedition.org/rbnu/1712 ; DOl : https://doi.org/10.4000/rbnu.1712

\section{cc) (†) (2)}

La Revue de la BNU est mise à disposition selon les termes de la Licence Creative Commons Attribution - Pas d'Utilisation Commerciale - Partage dans les Mêmes Conditions 4.0 International. 


\section{Les bruits de la bataille et le silence des archives : cinq réflexions et un épilogue}

Ce texte est la traduction, inédite en français, d'une conférence prononcée dans le cadre de l'inauguration de l'exposition August 1914. Literatur und Krieg, qui s'est tenue du 16 octobre 2013 au 30 mars 2014 aux Archives littéraires allemandes de Marbach. Il s'agissait là du premier élément de la trilogie "Guerre et archives ", qui s'est poursuivie ensuite à la Bodleian Library d'Oxford pendant l'été, avec l'exposition Personal Stories from Downing Street to the Trenches, et s'achève à Strasbourg avec l'exposition 1914, la mort des poètes. L'année de commémoration du déclenchement de la Première Guerre mondiale aura donc été particulièrement fertile en partenariats pour la BNU et pour La Revue : après l'inédit, provenant lui aussi de Marbach, de Lola Landau publié dans le numéro 9, c'est au tour de cette réflexion sur un sujet peut-être moins souvent traité, celui de la perception des bruits de la guerre, d'apporter au public francophone un éclairage sur un thème de recherche par définition transnational, rejoignant les réflexions, hélas toujours actuelles, sur les séquelles psychologiques des soldats comme des civils dans les pays en guerre. La première édition allemande de ce texte paraît elle aussi en novembre 2014, dans le numéro 58 du Jahrbuch der Deutschen Schillergesellschaft.

\section{1 - Les psychiatres contre la littérature de guerre.}

En 1929, le ministère impérial berlinois du Travail réunit un groupe d'experts dans le but de constater si certains troubles nerveux peuvent encore être considérés comme étant des séquelles de la guerre ${ }^{1}$. De l'avis des experts, il est quasi impossible dix ans après d'établir un lien de cause à effet entre ces névroses et la guerre. Des études statistiques ont permis de conclure que les névroses dues à la frayeur causée par les explosions d'obus ne pouvaient plus avoir de signification dans la pratique. Les responsables d'entreprises de transport témoignent qu'on ne voit plus de ces " malades pitoyables qui tremblent de tous leurs membres et sont donc incapables de tout travail $»^{2}$. De même, la direction des services postaux confirme que ces " troubles nerveux par suite de frayeurs extrêmes " ont pratiquement disparu. Que voulait le ministère du Travail ?

Il s'agissait pour lui de voir si, dix ans après la fin de la guerre, il pouvait encore être question de dédommagements, question à laquelle devaient répondre tous les directeurs de centres neurologiques, experts en psychiatrie et juristes. Les experts rassurent le ministère. Ils déclarent presque unanimement que les événements traumatisants vécus pendant la guerre ne peuvent s'encapsuler dans le psychisme de l'individu. Certains psychiatres font toutefois remarquer que "le mécanisme hystérique une fois enclenché " par des faits de guerre peut continuer de fonctionner par pur automatisme, même s'il ne peut plus être relié directement à cet événement déclencheur ${ }^{3}$.

Onze ans après la fin de la guerre, les experts sont donc d'accord pour déclarer que, même si elle fut à l'origine provoquée par le fait " de se voir livré à un danger mortel sans aucun moyen de s'en défendre " ${ }^{4}$, une névrose ne peut plus être rattachée au conflit. La frayeur du choc originel, selon eux, s'est définitivement estompée. Elle n'a laissé de trace ni dans le corps ni dans le psychisme. De quoi donc alors la mémoire pourrait-elle se nourrir?

Que ni le corps ni le psychisme ne gardent trace de cette frayeur est une chance, disent les médecins. C'est pourquoi ils considèrent comme une "infamie " que la littérature et la photographie s'avisent d'entretenir artificiellement le souvenir de cette frayeur initiale. Car de cette façon, elles prolongeraient la durée de 
survenue des symptômes chez les névrosés de la guerre et retarderaient leur guérison. Cette littérature serait dommageable à la thérapie. Car alors que l'organisme aurait depuis longtemps vaincu la blessure, la littérature et les autres médias nourriraient la mémoire du malade en lui rappelant constamment, comme l'explique l'expert juridique, le conseiller ministériel supérieur Knoll, " par le récit de situations semblables l'effrayant moment qu'il a vécu, ce qui aurait un effet paralysant sur son activité ${ }^{5}$. Et comme le constate le Dr. Jossmann, assistant de Bonhoeffer à la clinique neurologique de la Charité, tout ceci pourrait avoir des conséquences absurdes : " car alors devraient être reconnus les droits à pensions de personnes ayant simplement assisté ou même ayant " pris part, vécu " le sinistre par la lecture des journaux; le motif d'une telle revendication serait alors que la simple vue ou la description crue qui leur aurait été faite de l'horreur aurait causé chez elles un tel choc, un tel traumatisme que par exemple elles ne pourraient plus prendre un train, donc exercer leur métier ${ }^{6}$.

Onze ans après la guerre, de l'avis des experts, il ne peut donc plus y avoir de lien entre les névroses et les choses vécues à l'époque. Mais c'est juste alors que commence à déferler la littérature de guerre. Les experts de 1929 pourraient bien avoir raison : le corps est un mauvais garant du souvenir de la douleur. C'est pourquoi les rituels symboliques et les médias comme la littérature, la photographie ou le film se devaient d'entretenir le souvenir de ces événements traumatiques. Voilà qui explique peut-être la soif de lecture d'un public nombreux, en 1929, pour cette littérature de guerre.

Mais ce que cette littérature est capable d'évoquer avec le plus de difficulté, et qui est sans doute l'élément le plus traumatisant au front, c'est le bruit.

\section{2 - L'oreille : point d'impact du traumatisme.}

Dans son livre No man's Land: Combat and Identity in World War I7, Eric Leed rapporte que les soldats étaient d'accord pour dire que ce qui causa les névroses, ce ne fut pas tant de voir exploser des substances chimiques que le vacarme assourdissant et la vibration du feu roulant de l'artillerie, endurés durant des journées entières. L'assourdissement provoquait une sorte d'état hypnotique qui ne put jamais être exprimé en paroles. Robert Graves confirme l'impossibilité d'exprimer par le langage le vécu acoustique. Pour les soldats du front, les per- missions furent des moments étranges et terribles, car ils se trouvaient au milieu de personnes qui n'auraient rien compris s'ils avaient essayé de raconter : et d'ailleurs ils n'auraient pas su raconter, " c'était impossible. On ne peut raconter le bruit, ce bruit qui ne s'arrêtait jamais... " ${ }^{8}$. En novembre 1914, Philipp Gibbs écrit : " Le vacarme était encore plus destructeur que l’idée de la mort proche. Il avait un effet atroce sur nous..." ". Un vacarme assourdissant venait des canons ennemis, " comme un constant roulement de tonnerre interrompu de temps en temps par de grandes secousses qui allaient se planter dans le cerveau et que le corps entier ressentait comme s'il se désagrégeait d'une façon effrayante $" 9$. Un éminent psychiatre militaire confirme cette expérience. Quelques mois à peine après le début des hostilités, les médecins militaires eurent à soigner une multitude de " surdités hystériques $"{ }^{10}$.

Une réalité sans substance pénètre dans le corps et dans le psychisme par l'oreille et le soldat n'a aucun moyen de s'en protéger. L'oreille devient le point d'impact $\mathrm{du}$ bouleversement traumatique. Si l'ouïe est bien le point d'impact essentiel des causes de " névroses de guerre ", la réaction d'une partie des psychiatres militaires semble en tirer les conséquences. Ils essaient d'atteindre par le conduit externe de l'oreille " les stèles de la mémoire qui y sont profondément enfouies ", comme l'exprime le psychiatre hongrois Ferenczi ${ }^{11}$. La fraction la plus dure des médecins essaie de remettre par d'autres chocs psychiques "sur les rails " ce qu'ils désignent comme " une innervation ayant subi une sorte de déraillement ". Ces thérapies sont en quelque sorte une prise d'assaut de l'oreille. Les psychiatres envoient des décharges électriques dans l'oreille externe, avec des injections salines, pour libérer à l'aide d'injonctions " proférées sur des tons de commandement " l'accès aux centres nerveux et annuler ainsi le "dérèglement psychopathique ". Par de telles " méthodes palliatives", on essayait d'empêcher que des soldats victimes de " dérangements moteurs hystériques ", ces soldats que l'on appelait les " trembleurs " ou les " agités nerveux ", ne retournent chez eux. Ils ne devaient pas être vus dans les rues de leurs villes comme des symboles vivants de la misère de l'armée. 


\section{3-Technique de survie : reconnaître les bruits.}

L'oreille étant le point d'impact principal des agressions psychiques, il faut donc éduquer le soldat à une méthode d'écoute qui soit une technique de survie. Les premières semaines au front, comme le raconte Ernst Jünger, sont une éducation de l'ouïe. "Peu à peu, il (le soldat) apprend à distinguer parmi les nombreux bruits ceux qui peuvent être dangereux pour lui ; il devine la trajectoire d'un projectile dès son premier tressaillement. Il apprend à connaître les moments et les lieux suspects et devient peu à peu un connaisseur des choses de la guerre qui se glisse aussi furtivement qu'un serpent à travers le terrain déchiqueté $[\ldots]^{12}$.

Mais Ernst Jünger lui-même ne maîtrise pas spontanément l'art de repérer les bruits. Le 25 avril 1915, il écrit encore : « Krach, Bautz! ssst ! ssst ! ssst - bum ! ». Une succession de sons plus ou moins bruts (Stephan Schlak) est censée rendre l'impression d'être sous les tirs. Les onomatopées et fragments de mots vont chercher dans des champs en marge du vocabulaire, proches d'un point zéro de la communication verbale, aboutissent même à une sorte de poésie dadaïste faite d'onomatopées comme " Bautz ! Hulululu " ou " Udja - Udja - Udja - klack ».

Huit mois plus tard, l'évocation des bruits obéit à un schéma un peu plus ordonné. Le 10 janvier 1916, Ernst Jünger propose une assez vaste typologie des ressentis auditifs. En voici quelques exemples :

— Un tir de fusil venant de loin ressemble à " une vive douleur au tympan".

— « Si on se trouve à l'endroit où le projectile va tomber par sa trajectoire naturelle, on l'entend assez longtemps qui se rapproche puis on entend le bruit de son impact, ce qui n'a pas d'effet particulier sur les nerfs, mais qui chez moi provoque involontairement un besoin de sourire ".

— « Des projectiles arrivant de flanc se reconnaissent à un certain sifflement provoqué par le fait qu'ils tournent sur leur axe horizontal ".

— L'obus de gros calibre, " c'est l'horreur ". "Si l'on se trouve loin du point d'impact, on entend un grondement dans l'air, comme le raclement d'un véhicule en marche. C'est pourquoi chez nous on appelle ces engins des ' corbillards', des 'omnibus', des ' malles de voyageur', etc. A la fin de leur trajectoire, un fracas terrible, déchirant ou parfois rien, quand ils n'explosent pas".

— Les grenades à main font " un bruit dans la tranchée qui se prolonge de façon étrange. C'est assez pour provoquer durant les heures qui suivent une sensation de nervosité incontrôlable ».
L'ouie prend l'exacte mesure du bruit, identifie la composition et le calibre du projectile d'après le son qu'il produit durant son vol et d'après l'explosion, et en mesure la distance. Le fait de décoder à temps les signaux acoustiques peut permettre au corps de réagir à la vitesse de l'éclair. Des soldats ayant eu une formation musicale, d'après Eric Leed, seraient parvenus à une véritable virtuosité dans l'art de reconnaître les différentes sortes de projectiles ${ }^{13}$.

Dans les tranchées, l'état d'alarme permanent est dû non seulement au fait acoustique, mais aussi aux vibrations du terrain et aux variations de la pression de l'air. Les très hautes fréquences, elles, ne passent pas par l'ouïe ${ }^{14}$.

Le soldat, dans cette situation, est pris entre deux certitudes contradictoires mais aussi inquiétantes l'une que l'autre : la présence audible d'un ennemi invisible qui le paralyse, et l'urgence de réagir très rapidement dès que le bruit s'estompe et que l'ennemi devient enfin visible en chair et en os. C'est une situation caractéristique que l'on trouve par exemple au début de la bataille de la Somme : l'ennemi anglais, invisible, s'est déjà introduit dans le territoire du soldat qui le sait et qui l'entend dans le crépitement du feu roulant, mais sa réalité corporelle reste encore dans un lointain indiscernable. Le soldat aux aguets sait que dès que les tirs cesseront et que le silence se fera, il verra l'ennemi se dresser au-dessus de la tranchée ${ }^{15}$. La paralysie motrice des soldats durant le pilonnage de leurs positions doit se muer en un clin d'œil en " promptitude à défendre sa peau " (John Keegan). Il leur faut alors, dès que le silence se fait, bondir hors de l'abri jusqu'au rebord de leur tranchée afin de tirer à la mitrailleuse sur l'ennemi.

Guetter l'élément étranger qui cherche à pénétrer dans ses propres lignes crée la peur si la possibilité de sortie est compromise. Si par contre il y a des possibilités de fuite ou d'esquive, on arrive plus ou moins à manœuvrer face aux menaces acoustiques et à l'attente panique que l'auteur de ce bruit se montre. On sait que le film d'horreur constitue un entraînement à réagir dans ce genre de situation. "Les meilleures histoires de fantômes, dit Ernst Jünger dans Eloge des voyelles, sont celles où l'on ne voit pas le danger approcher, mais où on l'entend " ${ }^{16}$. Il est donc possible d'apprendre à " gérer " les bruits de la guerre. Par contre cela n'est pas possible pour le fracas traumatisant d'une explosion d'obus. Les photos ou les films peuvent en montrer les colonnes de fumées, de boue, les corps déchiquetés. On peut les photographier. Mais le soldat anéanti par l'explosion n'a aucune aide à sa disposition pour parvenir à intégrer cet événement. 


\section{"Si on se trouve à l'endroit où le projectile va tomber par sa trajectoire naturelle, on l'entend assez longtemps qui se rapproche puis on entend le bruit de son impact, ce qui n'a pas d'effet particulier sur les nerfs, mais qui chez moi provoque involontairement un besoin de sourire ".}

\section{4-Comment la littérature de guerre, comment le langage rendent-ils compte de l'effet-bruit ?}

Que peut-on lire en août 1914 dans les journaux et les correspondances, qui sont, comme le remarque Heike Gfrereis, " comme les rouages d'une gigantesque machine à écrire mise en branle par cette guerre " ? Les archives montrent, dit-elle, "l'énorme énergie mise à écrire " par laquelle le soldat, dans son sentiment d'abandon, s'assure un reste d'autonomie sur ces petits bouts de papier. Mais entend-on le bruit de la bataille dans ces écrits archivés ? Le silence du document n'éteint-il pas le bruit pour attirer l'attention sur le vestige muet, sur les objets portant l'empreinte de la main, portés jadis sur le corps, ou envoyés dans des paquets venant du front ? $\mathrm{Ne}$ verrons-nous dans cette exposition ${ }^{17}$ que les témoins muets d'une époque?

L'écriture silencieuse peut faire mieux. Car elle est une réplique des sonorités de notre langage. En 1925 paraît un corpus rassemblant " les bruits d'armes et de projectiles de la guerre mondiale ". On y a rassemblé, à partir de récits et d'écrits de guerre allemands et français, le vocabulaire qui a servi à évoquer ces sons. On y trouve de longs inventaires de termes relatifs au bruit. Comme le phonographe, qui aurait pu fixer de manière plus ou moins fidèle le fait acoustique, n'existait pas encore, on a tenté dans les années vingt de voir dans quelle mesure la langue était capable de rendre la dimension sonore de l'expérience vécue alors. Cela semble possible surtout parce que le son ne nous parvient jamais comme un phénomène " purement " acoustique, et touche en général le corps tout entier. Comme le montrent les exemples rassemblés en 1925, le langage est capable par son pouvoir synesthétique de traduire le transfert d'énergie qui se produit de l'ouie vers les autres centres nerveux ${ }^{18}$.
— «S-sim comme une lame de couteau " (Frankfurter Zeitung, 1915, $\mathrm{n}^{\circ}$ 28, II.M)

- "Le crépitement fou des dents d'une mitrailleuse " (Ganghofer, 1915)

— « alors, loin en arrière, on entend s'approcher, gargouillant et hurlant, le premier gros calibre » (Kieler neueste Nachrichten, 1915)

— «[...] comme un train rapide déboulant d'un tunnel " (Der Völkerkrieg, 1915)

— « cela vient d'en face comme le cliquetis monotone de tasses ou d'assiettes dans une guinguette géante, pleine de clients bruyants " (Frankfurter Zeitung, 1916)

Les bruits de la guerre excitent simultanément plusieurs sens : la vue, l'ouïe, mais aussi le toucher, et opèrent par des images synesthétiques des rapprochements avec des expériences familières vécues en temps de paix (dans le dernier exemple notamment).

Ernst Jünger a essayé, dix ans après le conflit, de décrire "l'horreur " provoquée par l'expérience de l'explosion d'un obus, dans ses croquis surréalistes intitulés Le cour aventureux. Il amalgame le vacarme s'amplifiant qui fait perdre le sens de l'équilibre avec les vibrations qui traversent le corps de part en part et l'impression de sombrer dans un abîme sans fond.

"Il existe une sorte de grande plaque métallique très fine qui sert dans les petits théâtres à simuler le bruit du tonnerre. Imaginons un grand nombre de ces plaques plus fines encore et plus sonores, qui seraient superposées un peu comme les pages d'un livre, mais sans être pressées l'une contre l'autre, avec un espace ménagé entre elles. Je te soulève et te laisse tomber sur la plaque supérieure de cette masse et le poids de ta chute fait éclater la première plaque. Tu tombes et la deuxième plaque éclate plus bruyamment encore. Puis c'est la troisième, la quatrième, la cinquième plaque, et l'accélération de ta chute 
fait se suivre les détonations de plus en plus vite, ce qui ressemble à un roulement de tambour dont le tempo et la puissance iraient s'amplifiant sans arrêt. La chute et le vacarme allant s'accélérant produisent une sorte d'énorme roulement de tonnerre jusqu'au moment où finalement un terrible fracas fait voler en éclats les limites de la conscience ${ }^{19}$.

L'évocation surréaliste de Jünger est très proche du vécu. Elle puise son évidence dans le registre habituel du vocabulaire servant à décrire les phénomènes sonores. Le matériau réuni dans le corpus franco-allemand cité plus haut contient dès 1925 les trouvailles suivantes :

— «L'écho se prolonge comme l'effondrement d'une pile de plaques métalliques » (Frankfurter Zeitung, 1915)

— « Un terrible orage de tonnerre et d'éclairs, comme si la terre était une grande plaque de métal sur laquelle s'abattraient des volées de coups " (Frankfurter Zeitung, 1915)

- "C'était comme un géant qui aurait déchiré une gigantesque toile " (Völkerkrieg, 1915)

— « Des portes métalliques claquent sur leurs gonds, des portes blindées gigantesques " (Höcker, 1914)

La façon dont Jünger rend compte de l'horreur ressentie tire son évidence d'un corpus textuel où s'est rassemblé le vocabulaire dont se servaient les soldats du front, quand ils voulaient évoquer le bruit. Ceux-ci, de leur côté, se servaient de termes empruntés à la littérature d'épouvante (E. A. Poe et E. T. A. Hoffmann par exemple), termes consacrés qui étaient d'usage courant au $19^{\mathrm{e}}$ siècle pour évoquer l'horreur.

\section{5-Moyens techniques pour reproduire le fracas d'une explosion.}

Au stade où en était la technique phonographique durant la Première Guerre mondiale, ces moyens étaient tout à fait insuffisants. Siemens \& Halske concentraient alors leurs efforts sur des procédés de communication plus que sur des techniques d'enregistrement des sons. D'un appareil comme le phonographe, on espérait alors qu'il pourrait conserver et reproduire le son. Pourquoi ? Parce que cet appareil a moins de tâches à effectuer que le cerveau humain, qui est obligé, lui, d'affronter directement le bruit, d'en distinguer la provenance, de réfléchir aux façons de s'en défendre, alors que l'instrument ne serait compétent que pour l'enregistrement pur et simple du fait acoustique. Mais les enregistrements de l'époque, et ce jusque dans les années 20, furent très décevants. " La retransmission du son réel par l'appareil n'est qu'un bruit confus et ne crée nullement l'illusion... Un coup de fusil par exemple ressemble tout juste à une tape qu'on aurait donnée sur le microphone $"{ }^{20}$. Les ingénieurs du son se mettent donc à installer des laboratoires de bruitage afin d'obtenir des effets plus ou moins " authentiques".

Bien plus tard seulement, le film parlant américain parviendra à créer l'illusion du fracas traumatisant. Joris Ivens raconte comment son équipe de l'atelier Columbia de la CBS, en 1937, réussit par la synthèse de différentes pistes sonores à produire des sonorités crédibles pour son documentaire The Spanish Earth, alors que les sons originaux rapportés d'Espagne étaient complètement ternes et inutilisables.

« Tous ces bruits de guerre artificiels étaient basés sur ce que ma mémoire et celle d'Hemingway arrivaient à ressusciter. A Irving (qui était l'ingénieur du son principal), nous disions qu'une attaque aérienne ressemblait à des aboiements de chiens dans la nuit [...]. Les explosions d'obus, qui ne durent en réalité qu'un cinquième de seconde, nous les prolongions cinq fois plus. Le seul bruitage déjà utilisé auparavant fut un fragment d'enregistrement du tremblement de terre de San Francisco, mais que nous fîmes dérouler à l'envers, ce qui nous servit à rendre l'effet d'un bombardement $"{ }^{21}$.

Ivens fit écouter cette bande réalisée en laboratoire à des personnes qui avaient pris part à la guerre. Tous furent frappés par " l'authenticité " du son. D’ailleurs cette transposition "d'aboiements de chiens " en bruit de bombardement peut s'appuyer sur des formulations déjà enracinées dans la mémoire langagière. En 1915, la Frankfurter Zeitung écrit à propos d'une attaque de mitrailleuses : "C'était comme si tous les chiens de la ville s'étaient mis à aboyer en même temps $"{ }^{22}$. On trouve ailleurs (Der Krieg 1914-15) : "Combien de fois, en levant les yeux, nous pensions voir les hordes d'acier des chiens de guerre volant et sifflant au-dessus de nous, si proches étaient leurs hurlements et leurs aboiements ".

L'utilisation de l'enregistrement du tremblement de terre dans cette bande sonore demeure un événement acoustique unique pour le cinéma de l'année 1937. Ce n'est que bien plus tard, avec les installations Dolby-stéréo, et grâce à la possibilité d'amplifier à l'extrême les basses fréquences, qu'on put " immerger " le spectateur (Thomas Elsaesser parle d'" engulfment ") en provoquant une fusion de son ouïe avec les vibrations de son 
corps. Des instruments plus récents sont capables de faire vibrer le mobilier du cinéma, de telle sorte que les sensations acoustiques et optiques se transmuent en sensations tactiles ${ }^{23}$. Dans le film de Spielberg Il faut sauver le soldat Ryan, la séquence sur le débarquement en Normandie est un exemple limite d'une telle tentative de recréer par " engulfment " une situation traumatisante. Le langage en est-il capable?

Car il faut noter aussi que tous les écrits sur la guerre se virent soudain concurrencés par la photographie, ce " nouveau " médium qui fit de son côté l'inventaire muet des champs de bataille. En ce moment même, nous nous attendons à voir surgir des millions de photos numérisées, et leur nombre s'accroît sans arrêt. C'est elle, la photographie, et non la littérature, qui constitue désormais la voie royale pour la connaissance de la réalité de la Première Guerre mondiale. Elle semble nous rapprocher directement des réalités de la guerre car, comme le dit un jour Roland Barthes, la photographie dispose d'un pouvoir magique :

" D'un corps réel, qui était là, sont parties des radiations qui viennent me toucher, moi qui suis ici ; peu importe la durée de la transmission ; la photo de l'être disparu vient me toucher comme les rayons différés d'une étoile ${ }^{24}$.

Et les constructions langagières de la mémoire concernant la Première Guerre mondiale ? Ne nous touchentelles pas également comme la lumière d'une étoile lointaine?

\section{6 - Le pouvoir du langage}

Vers la fin de la République de Weimar, époque où le travail sur les expériences de la guerre fut complètement bâclé, le linguiste Karl Bühler parla du " désir jamais satisfait de dépasser le caractère indirect du langage et d'autres outils culturels ${ }^{25}$. Il entendait par là le besoin de l'être humain d'aller au-delà des contraintes et des limites de l'écrit. " Le désir de voir, le besoin d'un contact direct et de la communication des choses relevant des sens est un fait très compréhensible chez l'être doué de la parole. L'être humain qui a appris à lire et à exprimer par des mots le sens du monde, se voit tenu à l'écart par " l'intermédiaire langage " de ce que son œil sait boire, son oreille absorber, sa main saisir, et cherche à retrouver le chemin de cette magie, à faire s'épanouir dans sa totalité le monde réel en l'exprimant par des mots dans la mesure du possible ${ }^{26}$.
Bühler trouve comique que des écrivains essaient de s'approcher de la réalité avec des onomatopées. Celles-ci ne peuvent être que de " petits fragments égarés " dans le champ d'expression du langage. Il est d'ailleurs rare de retrouver dans les documents sur la guerre des tournures du genre :

" Pft-pft-pft, comme un moteur qui hoquète au démarrage " (Queri, 19)

" S sss itt, klatsch ! j'entends une balle atteindre mon voisin " (K 1915, $\mathrm{n}^{\circ}$ 143)

D'après Bühler, on pourrait peut-être, avec de tels phonèmes, évoquer " une succession de petites images sonores " pour satisfaire le besoin de " sons concrets " ${ }^{27}$, mais le langage a des possibilités bien plus riches. Celuici a en réalité le pouvoir de mettre les choses à distance par son caractère indirect et symbolique, qui obéit à son propre système de signes, et de les ramener ensuite à une proximité qui nous les rend accessibles. Alors que Bühler est sceptique quant à la possibilité pour le langage de " reproduire fidèlement " des données acoustiques par des phonèmes isolés, il loue par contre sa capacité à dépeindre des phénomènes sensoriels compliqués :

"Alors, comme un coup de couteau vif et soudain dans une table, claque la décharge d'un tireur d'élite " (Frankfurter Zeitung, 1916)

"Les projectiles explosent avec un bruit sourd, comme d'une bouche tenue fermée et que fait céder soudain le besoin de respirer " (Frankfurter Zeitung, 1915)

" Interminablement, comme un être qui n'aurait pas besoin de reprendre son souffle, le feu bouillonne et mugit " (Frankfurter Zeitung, 1916)

Bühler attribue un grand champ d'action à la dimension synesthétique du langage. Mais il reste sceptique quand il s'agit de croire que des phonèmes isolés tels que " ratsch ", " ssit ", " peng ", " rumms " et " klatsch " pourraient permettre d'accéder à des phénomènes acoustiques inconscients, comme des lucarnes qu'on aurait placées dans un environnement artificiel de symboles. Bühler s'élevait contre une tendance observée par Robert Musil quand ce dernier disait que les intellectuels de l'avant-guerre avaient la nostalgie d'un " vacarme métaphysique ". C'est possible... mais quand ce "vacarme" fut là, les plus intelligents d'entre eux en restèrent pétrifiés et ils n'eurent plus envie d'attribuer à ce traumatisme une dimension métaphysique. 


\section{7- Le silence des archives}

La question est de savoir si nous allons, en cette année de commémoration 2014, nous plonger dans cet océan de millions de photos comme dans un vacarme métaphysique, ou nous laisser aller dans le silence des documents écrits, sachant que la technique symbolique du langage nous permet de mettre une distance entre ces phénomènes et nous, et de nous en rapprocher d'autant plus par la réflexion, afin de les ressentir en pleine conscience.

Ainsi le silence de l'exposition de Marbach ne met pas à notre disposition un " résidu inerte " de l'écriture, ni une sorte de jardin du souvenir d'un "intervenant " nommé langage. Le silence règne vraiment dans les archives. "La lisibilité des signes est aussi fragile que leur matérialité ", remarque Heike Gfrereis dans son commentaire de l'exposition. "Plus on approche de l'écriture, plus elle peut se révéler inaccessible : on peut la toucher, mais pas forcément la lire ou la comprendre ". En l'espace d'un mois, en août 1914, on observe d'énormes évolutions : des documents venant d'artistes qui se lancèrent sans hésiter dans " l'engrenage de l'enthousiasme populaire ", qui admiraient "l'horlogerie de la guerre " où, comme l'écrit Armin T. Wegener le 9 août, "les rouages s'imbriquent et s'entraînent comme de véritables chefsd'œuvre " - le même Wegener qui voit neuf jours plus tard sur le front polonais une scène grotesque : " quand les soldats entassent les cadavres comme des harengs et qu'ils disent : ' encore un peu de moutarde par-dessus...', et puis défèquent dans la fosse creusée ", pour finalement, dès le 22 août, faire un bilan anthropologique qui anticipe ce que Sigmund Freud qualifiera plus tard de dérive de sous-humanité. Cette transformation a eu lieu sans bruit, mais l'écriture et la littérature peuvent aider à la reconstituer. En cela elles sont supérieures aux enregistrements sonores et aux photographies. Mais dans le silence des archives, vous tomberez sur quelque chose d'autrement évocateur que l'écriture : un vestige de la vie d'un jeune écrivain. Nous pouvons lire les lettres d'amour de Gustav Sack, qui fut mobilisé de force alors qu'il était opposé à la guerre, lettres qui vont d'août 1914 à décembre 1916. Lisons jusqu'au 26 décembre :

" Par courrier recommandé, Paula Sack reçoit le deuxième jour après Noël un petit paquet contenant les affaires que son mari avait sur lui quand il est tombé sur le front de Roumanie : une pipe avec des restes de tabac, un porte-monnaie et une petite boîte ornée d'un angelot contenant deux médaillons avec les photos de Gustav et de Paula, ainsi que la chevalière d'une corporation d'étudiants ".
Nous lisons cela tranquillement, comme une chose attendue : pas de doute, l'écriture est l'intermédiaire, les lettres d'amour fraient la route qui nous amène à cet objet dans la vitrine. Et puis l'horreur : la chose est là et déborde de loin l'écriture. Le petit paquet venu du front, à l'instar d'une relique, avec la magie de la présence d'une vie à jamais éteinte...

On comprendra que j'hésite, quand je parle de " relique ", à évoquer un autre objet qu'on nous présente là : le casque avec le trou de la balle mortelle que Jünger prit sur le cadavre d'un officier anglais pour sa collection de trophées. Mais si on en reste au dogme de la présence des objets, il faut accepter l'amoralité du contexte.

\section{8- Epilogue}

Dans l'état-major de l'armée autrichienne, on a essayé de voir, à l'aide de séquences de films muets, comment des explosions d'obus pouvaient provoquer des maladies post-traumatiques. Karl Kraus a relevé le grotesque de cette tentative des généraux de comprendre le traumatisme subi par le soldat du front dans son livre Les derniers jours de l'humanité :

"Quartier général. Salle de cinéma. Au premier rang l'archiduc Friedrich, commandant suprême des armées. A son côté, son hôte le roi Ferdinand de Bulgarie. On projette un film produit par la société Sascha qui présente exclusivement des images d'explosions d'obus de mortiers. On voit de la fumée qui monte et des soldats qui tombent. La scène se répète quatorze fois pendant une heure et demie. Le public de militaires regarde avec toute l'attention de connaisseurs. On n'entend pas un bruit. Mais chaque fois que l'obus de mortier produit son effet on entend au premier rang :

«Boumsti !!»

En Autriche, lorsqu'une poupée tombe d'un landau, une grue dans le tas de sable ou une tasse sur le carrelage, les enfants disent "boumsti ». Peu importe celui à qui Karl Kraus fait prononcer cette exclamation dans la salle de cinéma de l'état-major des armées - nous autres sommes un peu plus décents face à ces événements traumatisants. Mais sommes-nous plus doués en matière langagière que l'archiduc Friedrich ou l'un de ses généraux ?

\section{Helmut Lethen}

(traduction Françoise Bornemann) 


\section{Notes}

1 - Die «Unfall- (Kriegs-) Neurose ». Vorträge und Erörterungen gelegentlich eines Lehrgangs für Versorgungsärzte im Reichsarbeitsministerium vom 6.-8. März 1929, in Arbeit und Gesundheit. Schriftenreihe zum Reichsarbeitsblatt, 13 (1929)

2 - Ibidem, p. 48

3 - Ibid., p. 109

4 - Ibid., p. 17

5 - Ibid., p. 93

6 - Ibid., p. 36

7 - Leed, Eric, No man's Land : Combat and Identity in World War I, Cambridge, 1979, p. 126

8 - Ibidem

9 - Gibbs, Philipp, Im Granatenfeuer, in Frankfurter Zeitung und Handelsblatt, 27.11.1914, $\mathrm{n}^{\circ} 329$, première édition du matin. Cité d'après Encke, Julia, Augenblicke der Gefahr. Der Krieg und die Sinne 1914-1934, Munich, 2002, p. 175. Thèse non publiée. Ce travail contient la plus intéressante documentation sur la guerre comme "école de l'écoute ".

10-Gaupp, Robert, Schreckneurosen und Neurasthenie. Cité d'après Komo, Günter, "Für Volk und Vaterland ». Die Militärpsychiatrie in den Weltkriegen, Münster/Hambourg, 1992, p. 71.

11 - Cité d'après Roth, Karl Heinz, Die Modernisierung der Folter in den beiden Weltkriegen. Der Konflikt der Psychotherapeuten und Schulpsychiater um die deutschen "Kriegsneurotiker"1915-1945, 1987, p. 18.

12 - Jünger, Ernst, Das Wäldchen 125, in Sämtliche Werke, p. 331 et suiv.

13 - Leed, op. cit., p. 124

14 - Voir à ce sujet la riche documentation chez Encke, op. cit.

15 - Keegan, John, Das Antlitz des Krieges, Francfort-sur-le-Main, New York, 1991, p. 269-280

16 - Jünger, Ernst, Lob der Vokale, p. 19

17 - H. Lethen fait ici bien sûr allusion à l'exposition August 1914. Literatur und Krieg.

18 - Les exemples qui suivent sont tirés de l'ouvrage de Dietrich Behrens et Magdalene Karstiens, Geschütz- und Geschosslaute im Weltkrieg. Eine Materialsammlung aus deutschen und französischen Kriegsberichten, Giessen, 1925. Les sigles entre parenthèses : FZ = Frankfurter Zeitung / I.M., II.M. = respectivement première et deuxième édition du matin : Ga III = Ganghofer, L., Die stählerne Mauer, Berlin, Vienne, 1915 ; Ga IV = Ganghofer, L., Der russische Niederbruch, Berlin, Vienne, 1915 ; Gen. = Frankfurter General-Anzeiger ; H = Höcker, P. O., An der Spitze meiner Kompanie, Berlin, Vienne, 1914 ; K = Kieler Neueste Nachrichten ; Kr I = Der Krieg, illustrierte Chronik des Krieges 1914/15, Stuttgart, $1915 ; \mathrm{M}=$ Morgenblatt der Frankfurter Zeitung ; Queri = Queri, Georg, Die hämmernde Front, Berlin, 1916; VV = Der Völkerkrieg, éd. par C. H. Baer, Stuttgart, 1915, vol. V ; W = Wertheimer, F., Im polnischen Winterfeldzug, Stuttgart, 1915.

19 - Jünger, Ernst, Das Abenteuerliche Herz. Aufzeichnungen bei Tag und Nacht. Erste Fassung (1929), Stuttgart, 1987, p. 15 et suiv.

20-Kesser, Hermann, Bemerkungen zum Hör-Drama, in Die Sendung, ${ }^{\circ} 29$ (1931). Cité d'après Schneider, p. 189.

21 - Ivens, Joris, Die Kamera und ich. Autobiographie eines Filmers, Reinbeck, 1974, p. 99

22- Du front de l'Isonzo sont parvenus des propos du même type : on évoquait des chats, des chiens, des écroulements selon les différents engins meurtriers. Les projectiles de l'infanterie étaient en général évoqués par des gazouillis, des sifflements et des claquements au moment de l'impact (propos rapportés par Lutz Musner).

23- Flückiger, Barbara, Sound Design. Die virtuelle Klangwelt des Films, Marburg, 2001, p. 208 et suiv.

24-Barthes, Roland, Helle Kammer (= La Chambre claire), p. 9o. Référence dans l'édition française : p. 854, par. 34 dans Euvres complètes, tome V, nouvelle édition revue et corrigée, Paris, 2002.
25 - Bühler, Karl, Sprachtheorie. Die Darstellungsfunktion der Sprache, Jena, 1934, p. 195

26 - Ibid., p. 195

27 - Ibid., p. 203

28 - Kraus, Karl, Die letzten Tage der Menschheit

29 - Rapporté par Ralf Bogner. 